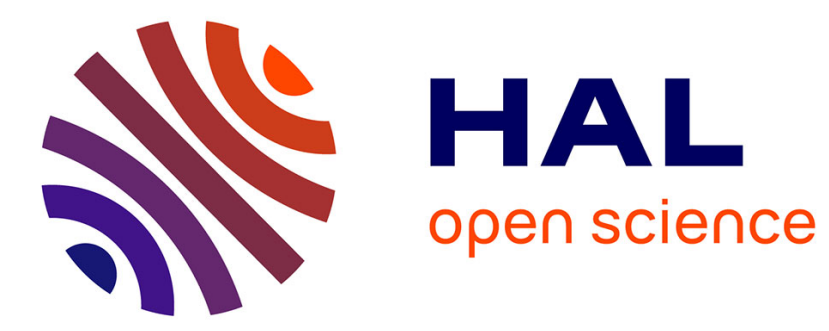

\title{
Les problèmes de l'échelle dans les céramiques grecques à reliefs
}

Gérard Siebert

\section{To cite this version:}

Gérard Siebert. Les problèmes de l'échelle dans les céramiques grecques à reliefs. KTÈMA Civilisations de l'Orient, de la Grèce et de Rome antiques, 2004, 29, pp.17-28. halshs-00003820

\section{HAL Id: halshs-00003820 \\ https://shs.hal.science/halshs-00003820}

Submitted on 4 Feb 2005

HAL is a multi-disciplinary open access archive for the deposit and dissemination of scientific research documents, whether they are published or not. The documents may come from teaching and research institutions in France or abroad, or from public or private research centers.
L'archive ouverte pluridisciplinaire $\mathbf{H A L}$, est destinée au dépôt et à la diffusion de documents scientifiques de niveau recherche, publiés ou non, émanant des établissements d'enseignement et de recherche français ou étrangers, des laboratoires publics ou privés. 


\section{Extrait de KTEMA 29, 2004, p. 17-27.}

\section{Les problèmes de l'échelle dans les céramiques grecques à reliefs}

RÉsumé. - La céramique à reliefs partage, pour ce qui est de l'échelle de la représentation, un certain nombre de règles communes avec la peinture de vases. Tant que jouent la loi du cadre et celle de la soumission du décor à la forme, des proportions cohérentes existent entre les figures. Tel est notamment le cas pour les frises modelées des vases attiques à reliefs polychromes. Mais la technique de fabrication au moule conduit à une organisation paratactique des frises et à la perte des repères spatiaux. Les fonds décorés de côtelures isolent les appliques dans un espace fictif. Extraites de leur ancien contexte narratif et réemployées en motifs isolés, des figures se transforment en signes, hors espace et hors échelle, sur des céramiques funéraires étrusques et apuliennes. L'histoire des bols à reliefs hellénistiques montre la déperdition progressive de l'échelle, depuis les séries homériques - dont les parois inscrites supprimaient déjà l'effet de profondeur - jusqu'aux vases purement ornementaux. L'utilisation de poinçons crée alors des échelles préfabriquées dans un univers décoratif sans espace.

ZuSAMMENFASSUNG. - Reliefkeramik hat, was den Maßstab der Darstellung betrifft, mehrere Grundprinzipien mit der Vasenmalerei gemeinsam. Solange der Dekor der Form unterstellt bleibt und der Fries in den Bildraum streng eingefaßt ist, werden auch die Figuren durch passende Proportionen miteinander verbunden. So ist es, z. B., der Fall mit der attischen polychromen Reliefkeramik, deren freihändig modellierten Figuren sich von den gemalten nicht grundsätzlich unterscheiden. Herstellung mit Hilfe von Matrizen (Plakettenvasen, Applikenkeramik), um so mehr Massenfabrikation in gepunzten Formen, führen dennoch zu parataktischen Bildkompositionen mit dem Verlust des thematischen Zusammenhangs und jedes räumlichen Anhaltspunktes. In dieser raumlosen, allein von Ornamentik beherrschten Bilderwelt, welche Mensch, Gebäude, Pflanze auf die gleiche Ebene stellt, kann kein Maßstab mehr richtig funktionnieren. Frühere Bildelemente können nun als Einzelmotive in abstrakte Zeichen ausarten - so öfters in der etruskischen und apulischen Sepulkralkeramik-. Standartisierung der Punzen befördert eine Art Fertigmaßstab, wie bei den späteren megarischen Bechern.

Après les publications récentes dont les céramiques à reliefs de l'Antiquité ont été l'objet, il est possible de mieux répondre aux questions archéologiques et historiques que posent ces catégories de vases*. Qu'il s'agisse des pithoi archaïques grecs, du bucchéro étrusque, des bols mégariens hellénistiques ou des sigillées romaines, les connaissances ont progressé sur les techniques de

*Principales abbréviations:

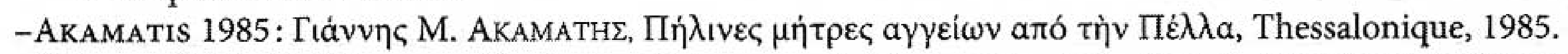

-HÜBNER 1993: G. HÜBNER, "Plastischer Dekor an griechischer Keramik. Gestaltungsprinzip und formaler Stellenwert", JdI 108, 1993, p. 321-351.

-Michetтr 2003: L.-M. Michetti, Le ceramiche argentate e a rilievo in Etruria nella prima età ellenistica. Acc. Lincei, Mon. Ant. , Ser. Misc. VIII (= Ser. Gen LXI), 2003.

-Sinn 1979: U. Sinn, Die homerischen Becher. Hellenistische Reliefkeramik aus Makedonien, AM, 7. Beiheft 1979

-Zervoudaki 1968: E. A. Zervoudaki, "Attische polychrome Reliefkeramik des späten 5. und des 4. Jahrhunderts v. Chr. ", AM 83 (1968), p. 1-88. 
fabrication, la localisation des fabriques et leur chronologie, l'utilisation et la commercialisation des produits ${ }^{1}$. Dans la mesure où la plupart des céramiques à reliefs comportent des décors figurés, elles ont également donné lieu à des enquêtes iconographiques. Beaucoup plus rares, en revanche, sont les études qui abordent leur imagerie d'un point de vue iconologique ou stylistique, si ce n'est pour traiter des modèles toreutiques dont les potiers se seraient plus ou moins librement servis ${ }^{2}$. Pourquoi ce blocage de la curiosité vis-à-vis d'interrogations que continue de susciter la peinture de vases? Le frein semble venir de l'opinion répandue, déjà partagée à certaines périodes de l'Antiquité, que les processus de fabrication et les exigences de la clientèle «chasse(nt) irrémédiablement (cette céramique) du cercle de l'art pour la confiner dans un artisanat déchu ${ }^{3} »$.

Mon propos sera d'interroger la céramique à reliefs, plus particulièrement ses productions hellénistiques - les plus visées par le blâme esthétique - selon un critère précis: celui de l'échelle de la représentation, applicable à toute image, quelles que soient sa technique de production et sa valeur artistique intrinsèque. L'échelle, c'est-à-dire la juste proportion des figures entre elles et, globalement, par rapport à la réalité, donnait leur cohérence visuelle aux compositions d'époque archaïque et classique, sur les vases décorés comme sur les reliefs en marbre. Encore faut-il se souvenir qu'une image peinte ou sculptée ne reproduit jamais les relations de grandeur observées dans la nature. En outre, dans l'art anthropocentrique grec et selon un a priori culturel, l'échelle est fournie par la figure humaine. Ce sont là les deux principales raisons - la construction idéale des images et la référence à l'homme, mesure de toutes choses - qui se trouvent à l'origine des principales "disproportions» que l'on constate en permanence dans l'imagerie hellénique. Elles correspondent à des codes de figuration dont traitent dans ce volume d'autres contributions à l'étude de l'échelle.

\section{Principe structurant et échelle d'harmonie}

Une très ancienne utilisation du décor plastique sur les vases grecs correspond au "principe structurant ", bien mis en lumière par G. Hübner ${ }^{4}$. La recherche de la juste proportion concerne alors non pas la relation entre plusieurs figures du décor, mais celle qui existe entre l'élément plastique (en ronde bosse ou en relief) et tout à la fois la taille et la forme du support. Il ne s'agit pas, en l'espèce, d'un rapport de grandeur réel, mais d'une échelle d'harmonie. Sur des pyxides de style géométrique la poignée composée de petits chevaux s'ajuste de manière visuellement satisfaisante au récipient. Il en va de même quand, sur un cratère du Peintre de Cesnola, le bouton du couvercle prend la forme d'une hydrie miniature ou quand la lèvre d'un vase est garnie d'une couronne d'oiseaux ou de l'ondulation d'un serpent. A l'époque orientalisante le col et le bec verseur de certaines oenochoés affectent l'aspect d'une tête de griffon ou d'oiseau. Encore dans la céramique classique, la tige ou l'anse d'une coupe peuvent prendre une forme phallique, l'embout d'un épinétron, celle d'un buste de femme ${ }^{5}$. Ce que j'appelle l'échelle d'harmonie est d'autant

(1) L'ouvrage de F. Courby, Les vases grecs à reliefs (1922), donne une vue d'ensemble qui, pour l'essentiel, n'a pas vieilli. Des contributions nouvelles, en documents et en idées, sont périodiquement apportées par les congrès internationaux de

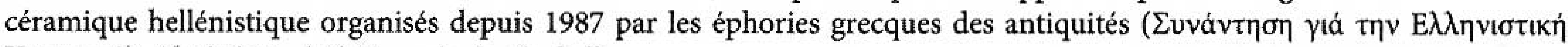
Kepauıкท́), Abrégé ici: ( $\left.\mathrm{n}^{\circ}\right)$ Congrès de cér. hell .

(2) De notables exceptions constituent, à cet égard, les études de G. HüBNER (bibl. ci-dessus) et, plus anciennement, celles d'U. Hausmann, en particulier Hellenistische Reliefbecher aus attischen und böotischen Werkstätten (1959).

(3) J.-P. MOREL, "Les céramiques de l'époque hellénistique en Italie: hellénisme et anhellénisme», Akten des XIII. Internationalen Kongresses für klassische Archäologie, Berlin, 1988 (1990), p. 161-171.

(4) HüBNER 1993, en particulier p. 322-327, 337-340, 347.

(5) Pour les exemples cités, on se reportera à E. Simon, Die griechischen Vasen (Hirmer 1981), pl. II, 7, 10, 14 et III, 21, 24, 216. - Le principe décoratif est différent lorsque, comme souvent dans le domaine ionien, le décor plastique se confond avec le corps même du vase: par ex. J. DucAT, Les vases plastiques rhodiens archaïques en terre cuite (1966). 
plus sensible au regard que le décor reste au service de la forme. On accentue par des moyens plastiques des points ou des zones-clefs, comme le fait la sculpture dédalique en soulignant chez l'Apollon de Manticlos les articulations du cou, de la taille, des genoux. Pour le potier, il s'agit de souligner la morphologie du vase, sans menacer son équilibre. La contre-épreuve est fournie par les céramiques du type de Canosa. L'échelle d'harmonie y est rompue parce que les statuettes greffées en guise d'anses ou de cols ou implantées sur le corps du récipient fonctionnent comme des pièces rapportées ${ }^{6}$.

\section{Décor peint et décor en relief: des règles communes}

Les principales techniques utilisées à l'époque archaïque pour le décor en relief sont d'une part le modelage à main libre, d'autre part l'emploi du moule et de la roulette ${ }^{7}$. Du point de vue de l'échelle de la figuration, les grandes scènes mythologiques modelées sur les amphores et les pithoi ne présentent, hormis une embryonnaire troisième dimension, aucune différence fondamentale avec les représentations des mêmes sujets par les peintres de vases. Sur le col de l'amphore de Mykonos, les dimensions du cheval de Troie sont réglées par une loi du cadre à laquelle sont également soumis les guerriers distribués dans l'espace de la métope selon les mêmes codes que dans la figure noire ${ }^{8}$ : les guerriers placés «au-dessus» de l'animal sont ceux de l'arrière-plan, mais l'arbitraire de la convention se manifeste dans le maintien de la même taille pour les assaillants représentés devant le cheval et aussi dans le surdimensionnement des épées qui, au premier plan, recoupent ses jambes. L'échelle fonctionne plus simplement dans les scènes d'ilioupersis de la panse, avec l'isocéphalie entre assaillants et femmes et avec la taille réduite des enfants massacrés. Sur les pithoi aux naissances divines sont mises en œuvre d'autres ressources ou imposés d'autres choix: hiérarchie symboliquement traduite par les proportions des figures; échelle dictée par un espace résiduel ${ }^{9}$. On retrouvera ces catégories dans les céramiques hellénistiques à reliefs.

L'estampage au moule, que F. Courby préfère appeler "poinçon en creux ${ }^{10}$, permet de réaliser mécaniquement les motifs répétés dans les bandes ornementales. Entre les listels qui les délimitent, guerriers, sphinx, chevaux, bouquetins paissant sont représentés en frises analogues à celles des vases peints géométriques et orientalisants. Le principe décoratif de l'itération supprime alors la question même de l'échelle, comme la supprime, pour des raisons inverses, l'isolement d'autres figures, plus monumentales, dans des cadres métopiques ${ }^{11}$. Le relief imprimé à la roulette offre l'avantage de pouvoir composer, comme le modelage libre, des scènes plus complexes, mais dans un registre miniaturiste. Il a atteint sa pleine expression dans le répertoire mythologique de l'atelier

(6) Pour un choix d'exemples caractéristiques: G. SIEBERT, «Des vases apuliens à figures rouges aux céramiques à décor polychrome et plastique», Ktèma 10 (1985), p. 23-25, pl. III-VI, fig. 5-14. Les céramiques de Canosa ont été publiées et étudiées dans de nombreux travaux de Fr. VAN DER WIELEN-VAN OMMEREN.

(7) L'incision et le poinçonnage constituent des techniques d'appoint pour les ornements subordonnés: J. SCHÄFER, Studien zu den griechischen Reliefpithoi des 8-6. Jahrhunderts v. Chr. aus Kreta, Rhodos, Tenos, Böotien (1957), pl. I,1.

(8) Cf. dans ce volume M. Halm-Tisserant, p. 13-14.

(9) K. SCHEFOLD, Frühgriechische Sagenbilder (1964), fig. 12-13 et 34-35. Pour le parallélisme entre figures peintes et figures plastiques modelées, cf. SCHEFoLD, op. cit., pl. 27 a-b et SCHÄFER, op. cit., pl. VIII-IX. - Echelle imposée par un espace résiduel: l'assise absurde d'un cavalier sur un vase à reliefs d'Hérakleion (ScHäFER, op. cit., pl. II,3); les cavaliers rapetissés sous les anses des amphores du Peintre Affecté (H. Mommsen, Der Affekter. Kerameus II, 1980, passim); la taille des personnages dictée par un cadre architectural: celle, réduite, du porteur de trépied sur la première marche d'un autel à degrés et miniaturisation des piliers hermaïques de la plate-forme, sous le "plafond " formé par le rebord du skyphos. Sous l'anse, le personnage est nanifié par rapport à l'aulète, au victimaire et au canéphore du cortège (A. MALAGARDis, «Deux temps d'une fête athénienne sur un skyphos attique ", $A K 28,1985$, p. 71-92.)

(10) Op. cit. (n. 1), p. 36.

(11) Ibid., pl. III, b; SCHEFold op. cit. (n. 9), pl. 15,b; SCHÄFER (op. cit. n. 6), pl. I,1 et V-IX. 
de Thasos, à partir du second quart du VI ${ }^{e}$ siècle ${ }^{12}$. La portée de la roulette - un développement d'environ $17 \mathrm{~cm}$ pour une hauteur de $4 \mathrm{~cm}$ - génère des frises comparables à celles des Petits Maîtres contemporains. Malgré la standardisation de l'outil de production, les unités narratives ne sont pas stéréotypées. Dans la scène du départ d'Amphiaraos (pl. I, fig. 1), le graveur a mis autant de soin que les peintres traitant du même sujet à distinguer par l'échelle les adultes du jeune garçon Alcmaion debout devant Eriphyle et du nourrisson Amphilochos dans les bras de sa nourrice ${ }^{13}$. Quant au cocher Bâton, surélevé dans la caisse du char, sa taille réduite est l'effet d'une contrainte, celle d'un espace résiduel, qui s'exercera dans toute la peinture de vases ultérieure ${ }^{14}$. En revanche, dans la séquence de la course de biges aux jeux funèbres de Pélias, c'est la valeur symbolique de l'échelle qui prime, quand le trépied destiné au vainqueur atteint la hauteur des attelages. Le même trépied est au contraire proportionné à la taille d'Héraclès dans la scène plus narrative du rapt delphique (pl. I, fig. 1) ${ }^{15}$.

L'emploi sporadique du relief dans la peinture de vases sévère est sans commune mesure avec le phénomène de style que constitue à Athènes, à partir de la fin du $\mathrm{V}^{\mathrm{e}}$ siècle, une nouvelle céramique à décor plastique polychrome ${ }^{16}$. Sur les vases les plus achevés, la combinaison de la figure rouge, du modelage et de matrices crée, dans l'emploi des échelles, un dénominateur commun entre peinture et relief. L'artiste esquisse sur la paroi, au trait ou à la gravure, les emplacements et les contours de ses personnages. Il en résulte une composition dont les figures modelées ou moulées fonctionnent dans le même espace et selon les mêmes rapports de grandeur que si elles avaient été réalisées dans la technique du dessin. Des finitions à la barbotine contribuent à l'unité de la scène. L'hydrie de l'agôn entre Athéna et Poseidon pour la possession de l'acropole est, à cet égard, exemplaire ${ }^{17}$. Sur un lécythe du Louvre (pl. I, fig. 2-4), l'assemblée éleusinienne, avec ses grandes figures divines assises ou debout - têtes en forte saillie, issues de moules séparés et ajustés aux corps modelés - $s$ 'inscrit avec une parfaite cohérence dans un espace scandé par des accessoires liturgiques et paysagistes ${ }^{18}$. Quand les reliefs sont formés de plaquettes moulées, découpées aux contours et distribuées dans la hauteur du champ, l'effet est analogue à celui que produit, avec la suppression des registres, la peinture de tradition polygnotéenne, en Grèce et en Grande Grèce. Le supplice de Marsyas, sur un lécythe de Naples ${ }^{19}$, montre des spectateurs à l'arrière-plan, le Satyre à genoux, sa tête à la hauteur de la taille d'Apollon, que couronne une petite Nikè, dont l'échelle réduite est celle de beaucoup de

(12) A. Coulie, "Les vases à reliefs thasiens de l'époque archaïque», BCH 124 (2000), p. 99-160.

(13) Ibid., p. 110-116, p. 134, fig. 6.

(14) Supra, n. 7 et 8.

(15) Coulie, p. 116-120, p. 125-128 et p. 134, fig. 7, 8 et 11. - J.-J. MAFFre, «Une représentation insolite de la dispute du trépied », Eirene 36 (2000), p. 43-53.

(16) ZeRvoudaki 1968. Sur le décor plastique à l'époque des pionniers et à l'époque sévère: ZervoudaKi 1968, p. 73-74; HÜBNER 1993, p. 327-328.

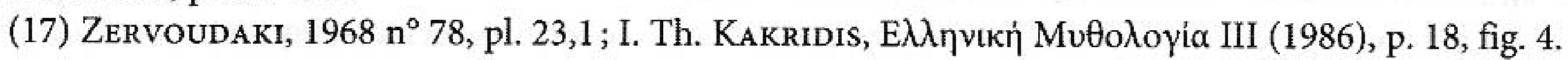

(18) ZERvoudaKi 1968, p. 16, nº 9, pl. 22, 1-2; H. METZger, Recherches sur l'imagerie athénienne (1965), p. 36, p. 45, $\mathrm{n}^{\circ}$ 16, pl. 15. - L'hydrie de Cumes au Musée de l'Ermitage (MetzGer, ibid., p. 40-41), n 36, pl. XX-XXII) pose, au regard de l'échelle de figuration, des problèmes qui seront évoqués plus loin. Les reliefs polychromes qui se détachent sur la glaçure noire sont modelés avec précision et les personnages sont proportionnés. Mais la frise, confinée sur l'épaule du vase, est soumise à la loi du cadre, délimité en haut par une couronne de laurier, en bas par les côtelures de la panse que traverse un étroit bandeau de lions et de griffons dorés.

(19) ZERVOUDAKI 1968, p. 18-79, nº 13 , fig. 2 et pl. 12,1.

(20) D'après les représentations littéraires, l’image mentale que l'on avait des figures allégoriques était incertaine. Eris, minuscule d'abord, est gigantesque à l'instant de se jeter dans la mêlée (Iliade IV, 440-445). Sur l'iconographique allégorique: H. A. Shapiro, Personifications in Greek Art. The representation of abstract concepts, 600-400 B.-C. (Akanthus, 1993); C. Aellen, A la recherche de l'ordre cosmique (Akanthus, 1994) - Pour des exemples de Nyx, Hypnos, Thanatos, Géras: cf., dans ce volume, M. Halm-Tisserant, p. 7 et pl. II. 
figures allégoriques dans la peinture de vases ${ }^{20}$. Un tel document annonce toutefois l'évolution vers un système décoratif à parataxe, même quand est préservée l'unité thématique des scènes.

\section{Du métal à l'argile}

Les céramiques de cette famille font l'objet d'un débat dont l'enjeu est considérable pour l'interprétation de l'échelle de figuration: c'est la question des relations entre potiers et toreutes. Les travaux récents insistent pour la plupart sur la créativité de l'artisanat céramique, en réaction contre l'idée reçue qu'un relief d'argile de bonne qualité est généralement obtenu par surmoulage d'un modèle en argent ou en bronze $\mathrm{e}^{21}$. A priori, trois cas de figure se présentent. Si l'intégralité d'un décor ou des sections entières d'un vase en métal ont été copiées mécaniquement, l'échelle du sous-produit aura la cohérence ou l'incohérence de l'original. $\mathrm{Si}$, au contraire, le potier utilise des matrices obtenues à partir d'originaux différents, les inégalités d'échelle qui peuvent en résulter sont recueillies telles quelles ou corrigées par des expédients que l'on décrira. S'il fabrique ses propres négatifs ou les fait réaliser par des graveurs à son service, il est seul maître des proportions qu'il entend introduire dans ses compositions.

On a toujours reconnu l'étroitesse des liens qui existent entre toreutique et céramique dans l'artisanat étrusque, un trait que J.-P. Morel qualifie d' " anhellénisme " ${ }^{22}$ et qui est sensible dans la morphologie des vases. Toute l'histoire du bucchero plaide en ce sens, bien qu'il faille raisonner, tant pour les formes que pour les décors, en termes d'interactivité des techniques plutôt que de simple imitation ${ }^{23}$. Les motifs circulaient et s'adaptaient à de nouvelles formes et à de nouveaux contextes. Probablement inspiré d'un modèle toreutique de Tarente, l'Héraclès entre deux Néréides d'un canthare étrusque de Berlin se retrouve (identique par la taille?) sur un relief de situle d'Arezzo. Mais le personnage est alors inséré dans un thiase, la léontè devient une draperie flottante et la massue, un flambeau ${ }^{24}$. Examinons, à travers quelques cas, les répercussions de l'inspiration toreutique sur l'échelle de figuration dans une catégorie de vases représentative de la koinè étrusco-italique: celle des vases argentés à reliefs, produits dans plusieurs fabriques entre le milieu du IV et le milieu du III ${ }^{\mathrm{e}}$ siècle $^{25}$. La reproduction, mécanique ou non, d'un modèle en argent ou en bronze est évidente sur des médaillons et sur de petites appliques d'anses. La difficulté de la composition circulaire favorise la copie à l'identique pour tel combat d'Héraclès avec le lion de Némée ou telle sacra conversazione entre le héros et Aphrodite en présence de Nikè ${ }^{26}$. Les recoupements des plans, caractéristiques d'un travail au repoussé, dissuadaient de procéder par extraits ou de modifier les proportions. Sur une attache d'anse de situle, les dimensions de la plaquette $(10 \times 12 \mathrm{~cm})$ permettaient de transposer le groupe formé par Socrate, Diotime et Eros tel

(21) Entre beaucoup d'autres prises de position, mentionnons celles de ZervoudaKi 1968, p. 74-75; de G. SIEBERT, Recherches sur les ateliers de bols à reliefs du Péloponnèse à l'époque hellénistique (1978), p. 211-215, avec bibliographie sur le sujet, p.417-419; de HüBNER 1993, p. 331-332; de MichetTI, p. 108-109; de J. BoARdMan ("Silver is white", RA 1987, p. 289296; réfutation des hypothèses de D. W. GILl et M. Vickers considérant la céramique à décor peint elle-même comme un succédané de la vaisselle en métal).

(22) J.-P. Morel, (supra, n. 3), p. 161-171. - Comparaison éclairante (p. 164-165 et fig. 2-3) entre le profil et la décoration d'un cratère en calice attique et ceux de son pendant dans la série de Malacena.

(23) J. Gran-Aymerich, «Le bucchero étrusque et les vases métalliques» dans Vaisselle métallique, vaisselle céramique (éd. J.-R. JANNOT), REA 97, 1995, p. 45-70.

(24) A. Greifenhagen, Beiträge zur antiken Reliefkeramik, JdI 21 ErgHeft (1963), p. 41-49, fig. 40-41, pl. I et III.

(25) Michetti, supra* : bibliographie p. 17.

(26) Fabrique de Volsini. MicheTtr, p. 60-61, n 297-298, pl. VI et LXVII; p. 57-58, nos 284 et 287, pl. LXVI. - Dès le milieu du IVe s., les petits vases à parfums ou à condiments font connaitre à la fois des reproductions exactes de modèles toreutiques et des copies simplifiées, souvent produites en masse: M. O. JENTEL, Gutti et askoi à reliefs étrusques et apuliens Leiden, Brill, 1976, passim. 
qu'il était sur le modèle en bronze ou en argent. En revanche, quand on intègre le même Socrate dans une frise de personnages sur un grand support de cratère ou sur un corps de situle, l'hétéroclite des modèles sollicités et leur présentation paratactique conduisent à la destruction de l'échelle ${ }^{27}$. En l'absence de listels ou de moulures réglant la hauteur des figures, celles-ci flottent dans un espace dépourvu de repères. Le potier ne cherche pas toujours à atténuer la gêne visuelle que le spectateur peut ressentir. Sur de grandes hydries falisques, couvertes d'appliques du col au pied, un pot-pourri de motifs puisés à divers cycles iconographiques, est superficiellement mis en scène par des adjonctions paysagistes. Les inégalités d'un terrain modelé à main libre y facilitent le voisinage entre une danseuse bachique nue et un combattant de la gigantomachie, entre un Héraclès dans sa grotte et une Nikè au bouclier. Mais les tableaux synoptiques du répertoire mettent en évidence les ruptures d'échelle. Un artifice plus sommaire encore consiste à aligner les personnages au-dessus $\mathrm{d}^{\prime} u$ ruban ondulé aux festons inégaux ${ }^{28}$.

\section{Images et signes. Syntaxe et parataxe}

En Attique, la production d'une céramique à reliefs polychrome était concomitante d'une crise du dessin, fondement de la peinture de vases traditionnelle. Mais les scènes, modelées ou moulées, y conservaient en général leur cohérence de sujet et d'échelle ${ }^{29}$. En Italie du Sud et plus particulièrement en Apulie, les vases funéraires, supports d'une imagerie eschatologique, connaissent une évolution différente. Les compositions tirées de la mythologie grecque survivent à l'état de débris dans une technique hybride. L'hippocampe, monture des Néréides qui portaient à Achille les armes commandées par Thétis, symbolise désormais à lui seul le voyage vers l'audelà. D'autres figures comme le char ou la Dame aux rinceaux deviennent des signes de félicité outre-tombe et cette nouvelle iconographie du signe, hors espace, ignore par définition la question de l'échelle ${ }^{30}$.

D'autres céramiques à reliefs, dont le principe décoratif n'est pas celui de l'image symbolique, peuvent se classer en fonction de la mise en œuvre syntaxique ou paratactique du répertoire. Les œnochoés ptolémaïques créées pour la fête des Arsinoei $a^{31}$ illustrent, avec quelques variantes, une liturgie royale: la libation que font les reines lagides sur un autel à proximité du bétyle d'Apollon Agyeus. Formée de trois ou quatre appliques, la scène est schématisée mais elle se situe dans un espace dont les éléments architecturaux sont plus ou moins proportionnés à la figure humaine. A l'inverse, les personnages des vases pergaméniens à reliefs appliqués, destinés à la célébration du culte attalide, défilent sur la paroi sans qu'apparaisse une règle de composition et sans que l'œil puisse toujours les mesurer. Certaines, il est vrai, ne sont pas dépourvues de repères, quand elles se trouvent associées à du mobilier ou à un embryon de paysage: une femme versant de l'eau dans une amphore, une déesse à l'arbre, Aphrodite au repos sur un trône ou un rocher, des amants sur un lit $^{32}$. Globalement, faute de syntaxe, les frises ne sont pas ancrées dans un espace perceptible.

(27) Plaquettes d'anse: Michetri, p. 169, n 140-141; p. 70-71, p. 167 et pl. LIV. Frises: Miche'tri, p. 70-71, $\mathrm{n}^{\circ} 3$; p. 142, 145 et 167 , pl. II et V.

(28) Michetti, Cat. n 457, pl. XCIV-CVI; p. 223-227, fig. 33-35 (motifs du répertoire); p. 144-145, nos 1-2, fig. 1 et pl. XIII-XVI.

(29) Supra, p. 20.

(30) G. SiEBERT , op. cit.. (n.6). La même iconographie du signe fonctionne dans le répertoire des vases étrusques.

(31) D. Burr Thompson, Ptolemaic Oenochoai and portraits in faience. Aspects of the Ruler Cult (1973).

(32) G. HüBNER, Die Applikenkeramik von Pergamon. Eine Bildersprache im Dienst des Herrscherkultes (Perg. Forsch. 7), 1993, Cat. nº $224-225$ (pl. 46-47), 236b (pl. 50), 136 sv. (pl. 27 sv.) 
Les mêmes manques, aggravés par la configuration de la paroi elle-même, caractérisent les vases côtelés à appliques (Plakettenvasen), dont les principaux ateliers ont été localisés à Alexandrie et en Crète ${ }^{33}$. Tandis que les cols des amphores ou des hydries et leurs bandeaux lisses à mipanse sont ornés de guirlandes en technique west-slope (sovraddipinta blanche et incisions), des appliques sont collées sous l'épaule, à même les côtelures. Les personnages ne sont pas découpés aux contours, comme les reliefs moulés de la céramique polychrome attique, mais se détachent sur un fond d'argile de forme ovale, dont les dimensions sont standardisées ${ }^{34}$. Un rythme est crée par la répétition des appliques alternant avec des panneaux côtelés, comme font les métopes avec les triglyphes d'une frise dorique. Sur l'hydrie de Bordeaux (pl. II, fig. 5-7 et pl. III, fig. 8), on ne voit aucun rapport entre l'Amazone combattant le griffon et le couple d'amoureux voisin. Sur l'amphore crétoise d'Inachôrion (pl. IV, fig. 9-10), isolés par les côtelures, Eros n'a plus de cible pour sa flèche et Héraclès frappe dans le vide ${ }^{35}$. Souvent les figures débordent sur la bande médiane, signe d'une échelle arbitrairement uniforme, inadaptée au champ et à la structure du vase. L'impression est celle d'un placage.

\section{Modèles et motifs. De la création à la production}

Le conflit entre une esthétique narrative et une esthétique décorative marque aussi l'histoire des bols mégariens. Selon les catégories et les séries, la question de l'échelle se pose très différemment. Sur les bols «homériques», fabriqués en Thessalie et en Macédoine ${ }^{36}$, les scènes inspirées de l'épopée et du théâtre, qu'elles soient réalisées par surmoulages ${ }^{37}$ ou librement sculptées dans des matrices, ont souvent une véritable cohérence thématique et spatiale. Tel est, par exemple, le cas pour les meurtres d'Agamemnon et de Cassandre, d'après les Nostoi d'Agias, ou pour l'épisode odysséen du supplice de Mélanthios ${ }^{38}$. Comme dans une frise de la peinture de vases, les personnages sont isocéphaliques, la hauteur étant réglée par les listels de la bordure et du médaillon (pl. V, fig. 11). Les distances entre les lieux (l'armurerie, la salle de banquet) et entre les adversaires (aux deux extrémités de la salle) sont suggérées avec une étonnante fidélité au texte $\left(\mathrm{Od}\right.$. XXII) ${ }^{39}$. L'unité de l'action est rendue par la gestuelle des acteurs et une justesse des proportions qu'un peintre de vases n'aurait pas mieux représentées. A l'inverse, dans la série vraisemblablement plus récente des moules de Pella, les capacités de figuration de l'imagier sont défaillantes, quand il veut illustrer l'épisode du sauvetage d'Enée par Poséidon (Iliade, XX, 325-329). Non seulement est perdue chez lui l'évocation de l'espace, si savante chez Homère, mais le groupe même du héros soulevé du sol

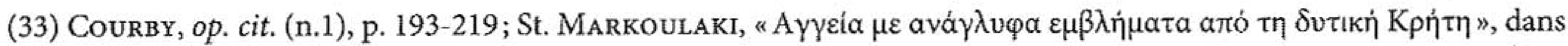
5 e Congrès de cér. hell., La Canée 1997; A. ENKLAAR, "La céramique fine hellénistique d'Alexandrie» et "Les vases côtelés à timbres» dans J.-Y. EMPEREur (éd.), Commerce et artisanat dans Alexandrie hellénistique et romaine (BCH Suppl. 33 ), 1998. p. 261-274.

(34) D'après ces indices, G. HüBNER 1993, p. 339, propose l'hypothèse de surmoulages à partir de harnachements décorés de plaquettes en métal.

(35) MARKoulaKi, op. cit. , p. 79-81, fig. 5-5a et 6-6a. Le couple des amants de Bordeaux se rencontre, sous des formes similaires sur des appliques de miroirs et des couvercles de pyxides: F. L. BASTET, BABesch 57, 1982,p.155-161, pl. 5-6; A. Schwartzmaeir, Griechische Klappspiegel, AM, 18 Beiheft (1997), pl. 20.

(36) U. SInN, Die Homerischen Becher. Hellenistische Reliefkeramik aus Makedonien, AM 7 Beiheft, 1979. Peut-être leur fabrication n'est-elle pas antérieure au premier quart du II siècle: S. Rotroff Gnomon 58, 1986, p. 474; C. RoGL, "Homerische Becher aus der Stadt Elis» dans $4^{e}$ Congrès cér. hell., Mytilène, 1997, p. 317-328.

(37) Des sections de la frise originale seraient alors imprimées dans des bagues, puis ajustées par le potier. Cf. U. HAUSMANN, op. cit. (n. 2), p. 34-36.

(38) Sinn, MB 36, p. 99, fig. 7; MB 21, p. 91, fig. 5. - M. Halm-Tisserant, "Autour du supplice de Mélanthios", Ktèma 20 (1995), p. 286-296, pl. I. (détails).

(39) L. Giuliani, Bild und Mythos. Geschichte der Bilderzählung in der griechischen Kunst, 2003, p. 264-269). 
par le dieu devient sur le poinçon un agglomérat dépourvu de toute échelle (pl. V, fig. 12-13). A la force expressive qu'ont quelquefois des groupes analogues chez les peintres (Ajax portant le corps d'Achille ou Enée portant Anchise ) se substitue une vignette naïve ${ }^{40}$.

L'échelle de figuration pose des problèmes particuliers avec les architectures ou les paysages qui peuvent former le cadre de l'action ${ }^{41}$. Les éléments paysagistes sont rares dans l'iconographie des bols à reliefs et se réduisent, par exemple, au rocher sur lequel sont parfois assis Paris ou Aphrodite $^{42}$. Dans une scène de combat au bord du Scamandre (pl. V, fig. 13), la forme humaine que revêt le dieu fleuve est bien intégrée à la grande forêt de roseaux qui couvrent sa rive ${ }^{43}$. L'anthropocentrisme impose au contraire sa loi lorsqu'il donne au couple d'Hélène et de Thésée, entrant triomphalement dans Athènes, la taille des remparts de la ville ${ }^{44}$. Peut-être l'intention est-elle moins claire dans nombre de scènes d'ilioupersis, par ex. pour le groupe de Priam et de Néoptolème atteignant le faîte du temple voisin (pl. VI, fig. 14). L'application mécanique de la règle du cadre suffit à expliquer la disproportion. En revanche, quand il donnait au Cheval de Troie la hauteur des tours, l'artiste renvoyait implicitement le spectateur à l'épisode du rempart abattu par les Troyens, la porte étant trop basse pour livrer passage ${ }^{45}$ (pl. VI, fig. 15). Un autre cas de figure correspond à une sorte d'invariant iconographique, puisqu'on le rencontre à des siècles de distance, aussi bien dans l'imagerie égéenne de l'Age du Bronze que sur les bols à reliefs hellénistiques. Quand sont représentés les défenseurs d'une ville postés aux créneaux ou les spectateurs sur les terrasses, seuls des bustes ou des têtes, énormes par rapport à la forteresse ou au palais, émergent derrière un mur. Il en est ainsi sur des fresques de Théra comme sur certains bols homériques de Pella. L'échelle fonctionne par juxtaposition ou superposition des hommes et des bâtiments, selon l'unique principe de la lisibilité ${ }^{46}$.

La méthode de fabrication des bols à reliefs portait en elle les germes d'un monde sans espace. Déjà les dernières séries homériques avaient perdu l'unité de lieu et d'action qui caractérisait les vases littéraires. Même quand la cohérence du récit est maintenue, comme sur des vases inspirés de l'Iphigénie en Aulide, on voit bien se dessiner un nouveau style. Sur le bol du Pirée ${ }^{47}$, seul le groupe

(40) Sinn, MB 7, p. 78-79, fig. 3, 2 (exemplaire de Berlin, acquis sur le marché d'art); AKamatis, p. 421, poinçon M75 et pl. 202, 292). Sur le groupe Enée-Anchise: G. Siebert, "Aeneas Flucht auf einer Beinschiene im Musée de la Ville de Haguenau », dans Praestant Interna. Festschrift für Ulrich Hausmann (1982), p. 289-294.

(41) G. Siebert, "Représentations d'édifices sur les bols hellénistiques à reliefs" dans Maquettes architecturales de l'Antiquité (Actes du Colloque de Strasbourg, déc. 1998). Travaux du CRPOGA 17, 2001, p. 409-423.

(42) G. Siebert, op.cit. (n.20), Cat. M 34 (pl. 25; cf. etiam pl.63) K 333 (pl. 39).

(43) Sinn, MB 7, p. 79, fig. 3, 2. Tel est aussi le cas sur quelques lécythes à fond blanc, notamment chez le P. des Roseaux, avec Charon, Hermès et des défunts au bord du Styx: $B C H 1$ (1876-1877), pl. I; AZ 1885, pl. 2; W. Riezler, Weissgrundige Attische Lekythen (1914), pl. 45; CVA Coll. Robinson, USA 7 (1938), pl. 43-46. - En règle générale le rapport d'échelle entre l'homme et la nature n'est pas celui qui s'exprime dans les fresques de la tombe étrusque de la Chasse et de la Pêche.

(44) SinN, MB 37, p. 99, fig. 7.

(45) AKamatis 1985, ns 297-298, pl. 169-175; n²29, pl. 176-178.

(46) Chr. Boulotis "Villes et palais dans l'art égéen du II ${ }^{e} \mathrm{M}$.» dans L'habitat égéen préhistorique (Colloque EFA 1987), BCH Suppl. XIX, 1990 , P. Darque et R. Treuil eds., p. 421-449. Cas de la «Master Impression» de La Canée, avec le gigantesque personnage au-dessus du palais ou de la ville (Boulotis, p. 434-437 et fig. 8). - A. FARnoux, "Image et paysage: l'exemple des fresques de la Maison Ouest de Théra» dans G. Siebert(éd.) Nature et paysage dans la pensée et l'environnement des civilisations antiques, Actes du Colloque de Strasbourg, Travaux du CRPOGA 14, 1996, p. 21-30, pl. I. - Akamatis 1985, Pella, Cat. n 301, fig. 15-16 et et pl. 182-183 - Pour des exemples dans la peinture de vases attique, $c f$. dans ce volume M. Halm-Tisserant, p. 12 et pl. X. Les exceptions n'en sont que plus remarquables. Sur une amphore campanienne du Peintre de Caivano, Capanée escalade le rempart de Thèbes sous le regard des défenseurs: tout y est correctement proportionné, conformément à une nouvelle esthétique illusionniste. (LIMC VII, 1994, s.v. "Contra Thebas", p. $139, \mathrm{n}^{\circ} 42$, pl. 544$)$.

(47) U. Hausmann, «Der Iphigeniebecher aus dem Piräus» dans Wandlungen. Festschrift E. Homann-Wedeking (1975), p. 220-235; SINN, $M B$ 53, p. 110-111, pl. I,1 et pl. 22-23. 
du char, avec l'arrivée de la famille dans le camp des Grecs forme une composition ordonnée aux éléments bien proportionnés. Les autres parties de la frise, sans trahir la lettre du texte, sont exécutées personnage après personnage, au moyen de poinçons imprimés séparément dans le moule. Le messager est représenté à échelle plus réduite que les figures royales, probablement pour signifier la hiérarchie. L'essentiel du changement qui s'amorce réside dans cette parataxe commandée par la technique des potiers. La production de masse, qui, dans l'ensemble du monde grec, bientôt banalise les bols à reliefs, joue de toutes les facilités du moule ${ }^{48}$. Même lorsque des répertoires de l'imagerie locale continuent d'être transcrits, comme à Athènes ou à Argos ${ }^{49}$, les scènes sont disloquées par le poinçonnage (pl. VII, fig. 16). Pour l'échelle de figuration, les conséquences sont d'autant plus importantes que le nouvel esprit décoratif favorise l'intrusion envahissante des motifs végétaux et la subordination croissante du récit mythologique aux ornements. Ce qui maintenant structure le décor, c'est le calice de lotus et d'acanthes: les dieux et les héros se trouvent relégués dans les intervalles, c'est-à-dire dans des espaces résiduels. L' échelle cesse de fonctionner selon les règles de la composition; elle cesse même d'être expressive et signifiante, puisqu'elle est devenue arbitraire et fortuite. Dans certaines productions tardives ou provinciales, tout sens des proportions est perdu. Eros peut n'être plus qu'une volaille accrochée à une touffe d'herbe ${ }^{50}$. Dès l'origine du genre, la circularité de la panse que n'interrompt sur le bol aucun élément morphologique (comme les anses verticales ou horizontales d'un skyphos) favorise le déroulement sans fin d'une ronde quasi immatérielle, où rien n'attire le regard ni ne crée la dimension et dont feuillages et personnages constituent les éléments également irréels ${ }^{51}$.

\section{La fin des images}

Ce qui représente le terme d'une évolution était annoncé dès les origines du genre. Si les bols mégariens les plus anciens sont, comme il semble, des vases à calices végétaux imités de prototypes en métal ou en faïence ${ }^{52}$, aucun problème d'espace ni d'échelle ne se posait à des artistes exclusivement soucieux d'harmonie décorative (pl. VII, fig. 17). L'introduction d'un répertoire figuré ambitieux ne changeait qu'en apparence la nature du décor. Certes, la mise en scène d'actions et de personnages exigeait une certaine cohérence narrative mais elle ne visait pas à la représentation du réel. En effet, sur les bols homériques, la réalité de l'espace est anéantie par l'insertion d'amples citations entre les groupes de personnages. La paroi du vase se fait livre, les textes étant distribués sur plusieurs lignes et colonnes d'écriture. La pratique de l'écriture sur les bols à reliefs homériques est constante, souvent, il est vrai, plus mesurée que dans la série représentée par le vase de Mélanthios. Un nom

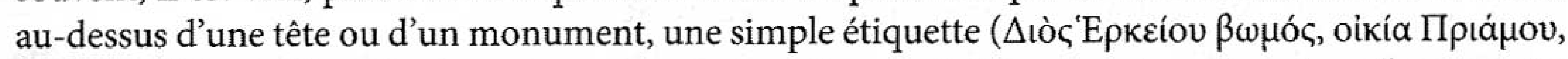

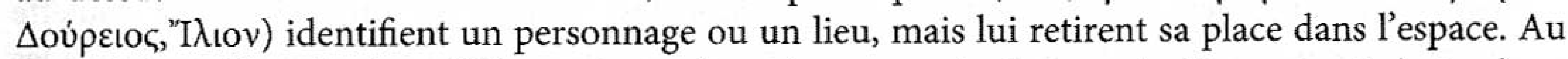
total, il s'agit d'une logique d'illustration, celle qui gouverne les images des livres et qui n'est qu'une variante de la logique décorative ${ }^{53}$.

(48) G. SIEBERT, «Les bols à reliefs. Une industrie d'art de l'époque hellénistique» dans P. LÉvêQue et J.-P. Morel (éds), Céramiques hellénistiques et romaines (Annales littéraires de l'Université de Besançon 242), 1980, p. 55-83.

(49) S.I. RotrofF, Hellenistic pottery. Athenian and imported moldmade bowls (The Athenian Agora XXII), 1982, p. 19-24; G. SiEBERT, "Thèmes troyens dans la céramique à reliefs hellénistique de l'Argolide» dans Iconographie classique et identités régionales (BCH Suppl. XIV), 1986, p. 57-65.

(50) S. KüNZL, Ein Komplex von Formschüsseln für Megarische Becher. Die «Mainzer Werkstatt» (2002), Cat. n 31-32, pl. 77-81.

(51) G. Hübner traduit cette impression du spectateur en évoquant «die flache Reliefhaut» (HüBNER 1993, p. 340).

(52) S. I. RotrofF, op. cit. , p. 6 sv.

(53) Des oeuvres plus ambitieuses que les céramiques à reliefs jouent toutefois de l'ambiguïté entre planéité et profondeur: Polygnote de Thasos utilisait l'écriture dans ses fresques de la Leschè des Cnidiens à Delphes. Des inscriptions parsèment les 
La standardisation des poinçons qui imprimaient le décor, évidente dans nombre de cas, engendrait l'uniformité des séries iconographiques, quelle que fût la combinatoire des motifs. D'une certaine manière ce processus créait une échelle, puisque l'échelle est la constance dans l'unité de mesure. Mais c'était une échelle préfabriquée, ne tenant aucun compte du contenu de l'image. Dans le sacrifice de Polyxène sur un bol du Musée National d'Athènes ${ }^{54}$ (pl. IX, fig. 18-19), l'eidôlon d'Achille est gigantesque. En position assise, la figure a même hauteur que le tumulus couronné d'une stèle. Il en résulte pour le poinçon de l'égorgement une double réduction de Néoptolème et de la victime, pour les mettre dans un rapport de grandeur plausible avec le tombeau. Ici l'échelle fonctionne dans le cadre du poinçon, mais au prix d'une nanification et d'une totale disproportion avec les autres personnages de la frise. Muni de sa boîte à outils garnie de jeux de poinçons, le potier était moins libre que le peintre qui, son pinceau à la main, pouvait, malgré beaucoup de contraintes et de conventions proportionner ses figures verticales, parfois faire mordre un casque sur un bandeau, composer à l'horizontale les grandes figures des héros morts et des géants endormis.

\section{Echelles et formules décoratives}

A travers l'histoire multiséculaire de la céramique à reliefs grecque, on observe, dans l'emploi de l'échelle de figuration, des constantes qui sont aussi celles de la peinture de vases. Elles tiennent, pour certaines à la structure même des supports de l'image. La loi du cadre, avec la partition du corps vasculaire en registres délimités par des frises ornementales ou de simples listels en relief, ne fait que traduire la soumission du décor à la forme. Tant que joue cette règle de l'artisanat céramique fonctionne aussi une échelle cohérente dans les représentations, mais modulée par un certain nombre de conventions:

- La mesure de référence est celle de la figure humaine, sur laquelle s'alignent paysages et architectures dans tous les arts figurés grecs.

- Des disproportions expressives, dans le sens du gigantisme ou du rapetissement, traduisent quelquefois la hiérarchie entre les personnages d'une scène.

- D'autres disproportions, ni expressives, ni significatives, sont dictées par les contraintes des espaces résiduels.

- Dans des séries comme celle des représentations eschatologiques sur les vases apuliens à reliefs polychromes, les figures devenues signes se situent hors espace, donc hors échelle.

L'espace conventionnellement construit selon la loi du cadre n'implique pas une formule décorative unique, ni l'uniformité dans la mise en œuvre de l'échelle:

- En frises continues, les personnages peints, modelés, moulés ou imprimés à la roulette entretiennent des rapports de grandeur cohérents à la condition qu'une syntaxe figurative les relie par les mouvements, les gestes, le sens. Quand, à la perte de l'unité thématique, s'ajoute la parataxe des unités iconographiques, l'espace lui-même est anéanti: faute de repères, l'échelle des personnages n'est plus sensible.

- L'espace, sans lequel il n'est point d'échelle, disparaît également lorsque les figures flottent sur la paroi ou sont enfermées dans des appliques répétitives sur un fond décoré.

compositions paysagistes des fresques de la Maison de l'Esquilin et de la mosaïque de Préneste. - Pour les citations de textes sur les bols homériques, on se reportera désormais aux analyses de L. Givliani (op. cit., n. 39, p. 272-280), qui démontre, contre $\mathrm{K}$. Weitzmann, que le «livre illustré» qui aurait servi de modèle aux potiers n'existait pas à l'époque hellénistique. Sur les bols homériques, la primauté revient aux images organisées en cycles. Sur certains vases, l'insertion de vers homériques, sous la forme d'extraits abruptement commencés et interrompus, se prête à un jeu littéraire, qui invite les banqueteurs à rivaliser de culture poétique en complétant les passages inscrits.

(54) Hausmann, op. cit. (n. 2), HB 25, pl. 36. 
- Avec la prédominance de l'esprit décoratif (et sa variante, l'esprit d'illustration) la question de l'échelle cesse de se poser. Devenus des motifs, comme les végétaux ou les ornements géométriques qui les entourent, les personnages ne connaissent plus d'autre loi que celle de l'harmonie visuelle. Harmonie toujours menacée par une technique de fabrication qui, par souci de productivité, use de surmoulages hétérogènes et de poiçonnages incohérents. Le nouvel outil du poinçon, souvent standardisé, génère une échelle préfabriquée.

L'échelle de figuration n'est pas une notion univoque. Pour comprendre son fonctionnement sur les vases grecs à reliefs, j'en ai tenté l'approche. par touches successives. En se fondant sur le matériel examiné ici, on ne saurait bâtir une théorie de l'échelle. La question est de savoir si, sur des bases élargies à l'ensemble de l'iconographie grecque, une telle construction est possible, à l'instar de la réflexion normative sur le canon en sculpture ou sur le module architectural. La conclusion qu'on tirera de la pratique des potiers est que l'échelle de figuration, à la différence du canon ou du module, est un concept empirique ${ }^{55}$.

Gérard SIEBERT,

UMR 7044,

Université Marc Bloch, Strasbourg II

(55) Sur les notions d'«échelle», de "proportion", de "canon», on consultera A. Souriau s.v. dans Vocabulaire d'esthétique (s.d. d'E. Souriau), PUF, 1990 Pour l'échelle appliquée à l'architecture, il suffit de renvoyer au texte de Vitruve au début du chapitre I du Livre premier, où est également développée la référence au module du corps humain: L'ordonnance d'un édifice consiste dans la proportion qui doit être soigneusement observée par les architectes. Or la proportion dépend du rapport que les Grecs appellent ảva oý́a ; et, par rapport, il faut entendre la subordination des mesures au module dans tout l'ensemble de l'ouvrage, ce par quoi toutes les proportions sont réglées Le corps humain a naturellement et ordinairement cette proportion... (VITRUVE, Les dix livres de l'architecture, III,1,1 Traduction de Claude Perrault, 1673, revue et corrigée par A. Dalmas, Ballarid, 1979), p. 301-305, 627, 1175-1178 - Sur les problèmes du canon dans l'art grec: E. BERGER, Der Entwurf, Bâle, 1992. 


\section{LÉGENDES DE L'ILLUSTRATION}

Fig. 1. Fragment de pithos. Musée de Thasos, inv. 2691 p. - A. Coulié, BCH 124 (2000), p. 111-112, fig. 9. Rapt du trépied de Delphes. Départ d'Amphiaraos. Photothèque de l'Ecole française d'Athènes (cliché $n^{\circ} 41295$ de J.-J. Maffre).

Fig. 2-4. Détail d'un lécythe à reliefs polychromes du Musée du Louvre, inv. CA 2190. Assemblée éleusinienne. H. Metzger, Recherches sur l'imagerie athénienne (1965), p. $36 \mathrm{n}^{\circ} 16$, pl. 15. Cliché P. Lebaube, Musée du Louvre.

Fig. 5-8. Hydrie à reliefs appliqués. Collection Universitaire de Bordeaux. - F. Courby, Les vases grecs à reliefs (1922), p. 210, D, fig. 33, 10-11 et pl. VII, c. Deux appliques avec variantes d'un combat entre Amazone et griffon. Sur l'une, l'Amazone frappe d'estoc, sur l'autre, de taille. Le croquis de F. Courby montre un bouclier au bras gauche de la seconde figure. Peut-être tient-elle la double hache. Sur chaque face de l'hydrie l'applique à l'Amazone alterne avec une autre image, qui représente un couple d'amants. Relief brouillé en certaines parties de la composition. La jeune femme dévêtue est appuyée contre l'homme assis sur un rocher, qui pose la main droite sur son épaule et la tête contre sa nuque. Le genou droit, au premier plan, et un pan d'étoffe oblique dissimulent le sexe. Dessin de M. HaIm-Tisserant, d'après des photographies que je dois à l'obligeance de Jean Marcadé.

Fig. 9-10. Amphore à reliefs appliqués. Musée de Kisamos inv. II12. Cliché de l'Ephorie de Crète, que je remercie en la personne de Madame Maria Andreadakis-Blazakis. Publication: St. Markoulaki, supra, op. cit. n.33

Fig. 11. Bol à reliefs. Staatliche Museen Berlin, inv. 3161 n. U. Sinn, Die Homerischen Becher (1979), p. 89-91, MB 21 et fig. 5,1. D’ après C. Robert, «Homerische Becher», 50. BWPr. (1890), p. 8, fig. A. - De gauche à droite la frise: a) Melanthios cherche des armes dans l'armurerie, avant d'être maitrisé par Eumée et Philoitios; b) à une extrémité du mégaron, Athéna encourage Ulysse et Télémaque, tournés vers les prétendants qui surviennent (figurés à l'arrière plan); c) pendaison de Mélanthios.

Fig. 12. Poinçon d'un moule de Pella ( $\mathrm{n}^{\circ}$ de cat. 310, p. 421, pl. 200). Sauvetage d'Enée par Poséidon. Dessin

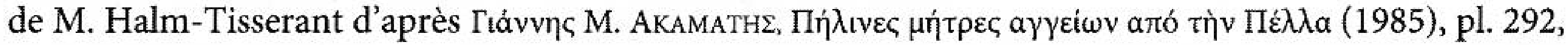
M. 75. Poinçon analogue sur le bol homérique de Berlin, inv. 30535: Sinn, op.cit. , p. 78-79, MB 7 (ici fig. 13).

Fig. 13. Bol à reliefs de Berlin, inv. 30535. De gauche à droite : a)Reconciliation entre Achille et Agamemnon en présence d'Ulysse; b) duel entre Achille et Enée, sauvé par Poséidon; c) duel au bord du Scamandre.

Fig. 14. Fragment de moule de Pella. G. Akamatis, op.cit., ${ }^{\circ}$ de cat. 297, p. 177, pl. 170. Scènes d'ilioupersis. Je remercie G. Akamatis de m'avoir libéralement autorisé à publier cette photographie et celle de la fig. 15, provenant de ses archives.

Fig. 15. Détail d'un moule de Pella. G. Akamatis, op. cit., $\mathrm{n}^{\circ}$ de cat. 299, p. 180 et pl. 176. Cheval de Troie et porte de la ville.

Fig. 16. Fragment de bol à reliefs d'Argos. Au-dessus d'un calice végétal bas, frise paratactique. De gauche à droite: jeune femme couronnant un trophée, trophée, Amymonè, Poséidon, Cassandre au palladion. G. Siebert, Recherches sur les ateliers de bols à reliefs du Péloponnèse à l'époque hellénistique (B.E.F.A.R. 233, 1978), pl. 23, M. 10. Photothèque de l'Ecole française d'Athènes (cliché E. Séraf).

Fig. 17. Fragment de bol à reliefs d'Argos. Calice d'acanthes et de lotus, avec fleurs d'aracées dans les intervalles. G. Siebert, ibidem, pl. 12, DI.1. Photothèque de I'Ecole française d'Athènes (cliché n ${ }^{\circ} 35726$; E. Séraf).

Fig. 18-19. Bol du Musée Archéologique National d'Athènes, inv. 14624 (U. Hausmann, Hellenistische Reliefbecher, pl. 36, HB 25). Je remercie pour cette photographie M. Nikolaos Kaltsas, Directeur du Musée. Sacrifice de Polyxène sur le tombeau d'Achille, en présence de l'ěl $\delta \omega \lambda o v$ du héros. Dessin du poinçon par M. HaIm-Tisserant. 
Planche I



fig. 1

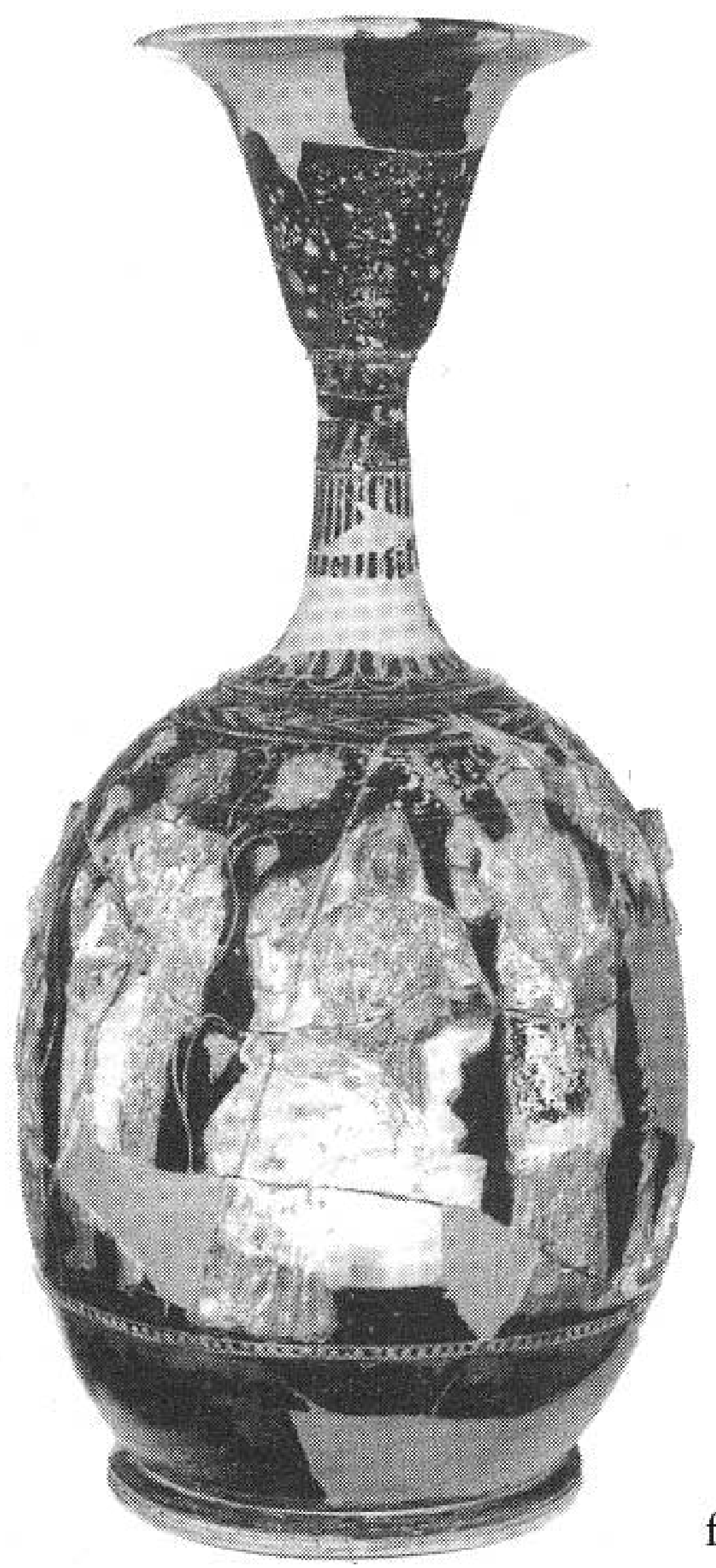

fig. 2





\section{Planche III}

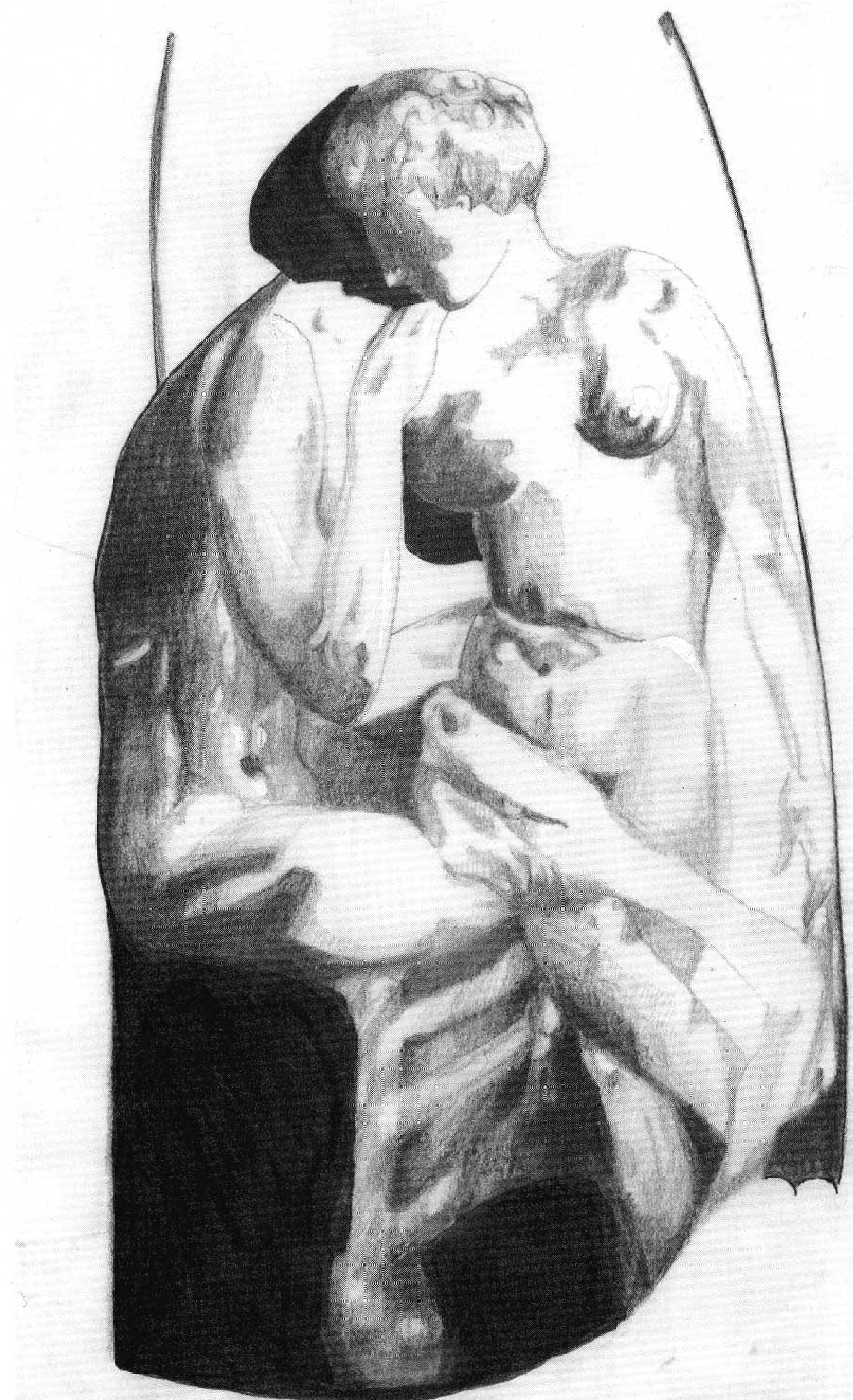

fig. 8 
Planche IV
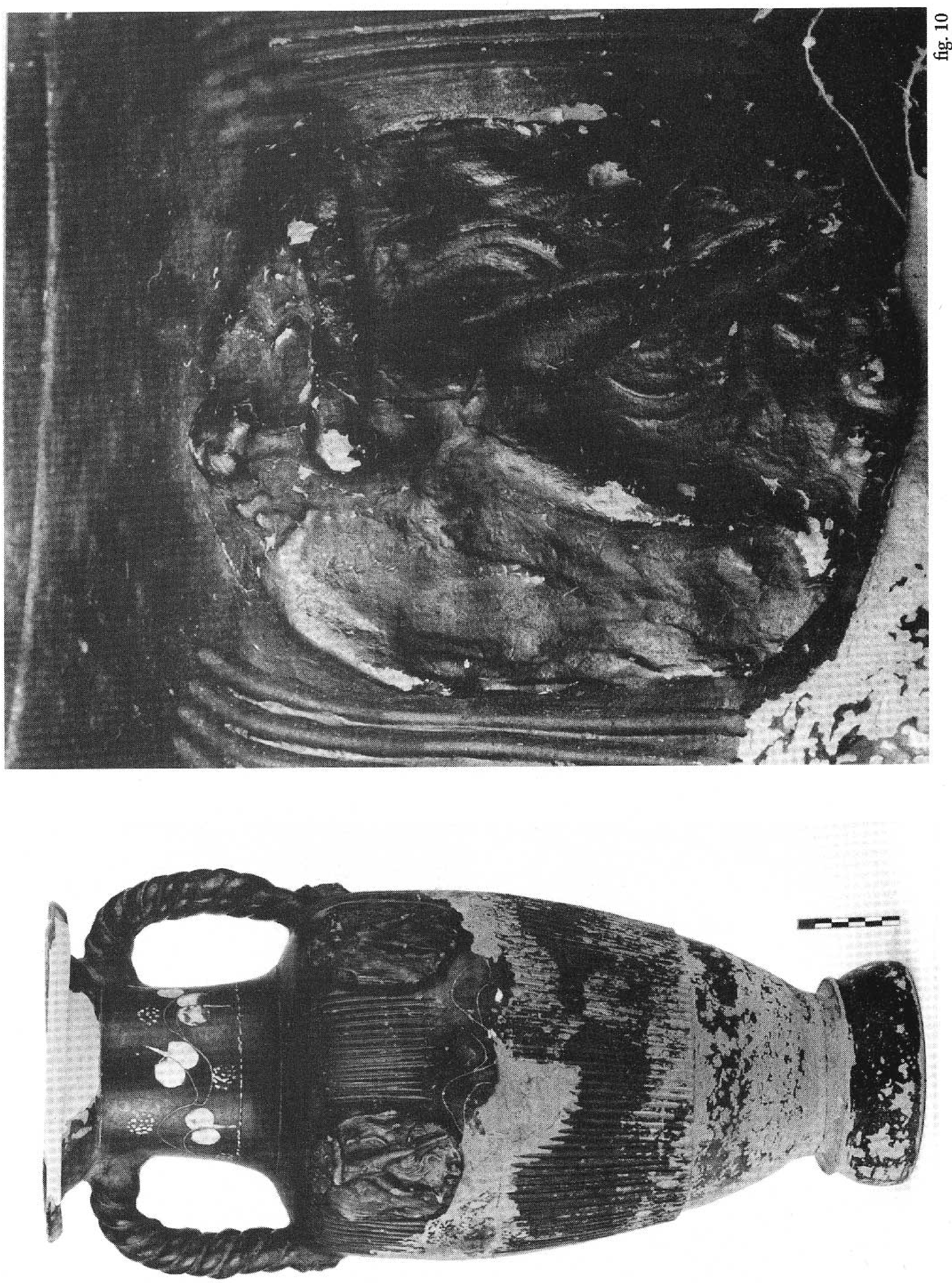
Planche V

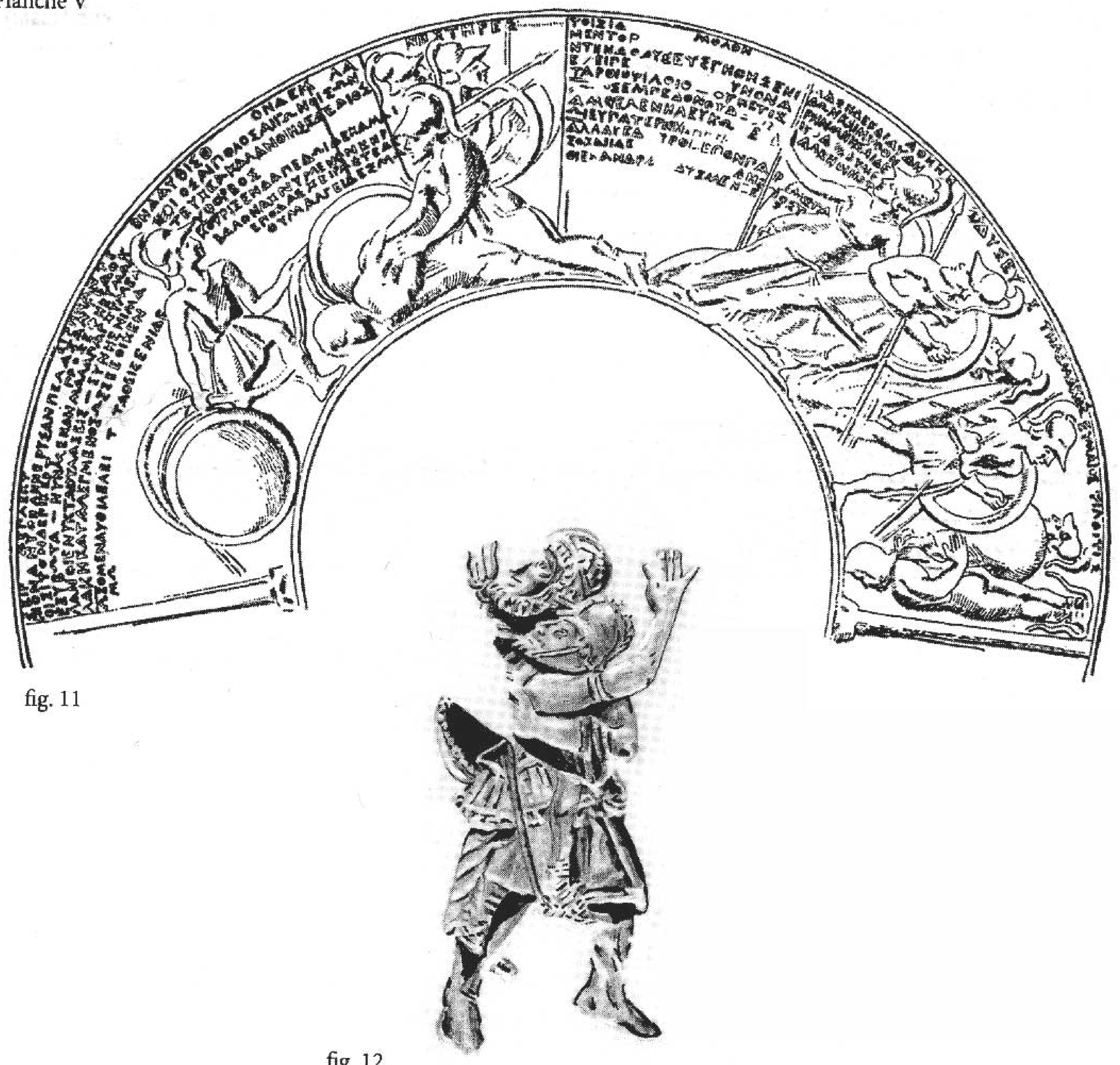

fig. 12

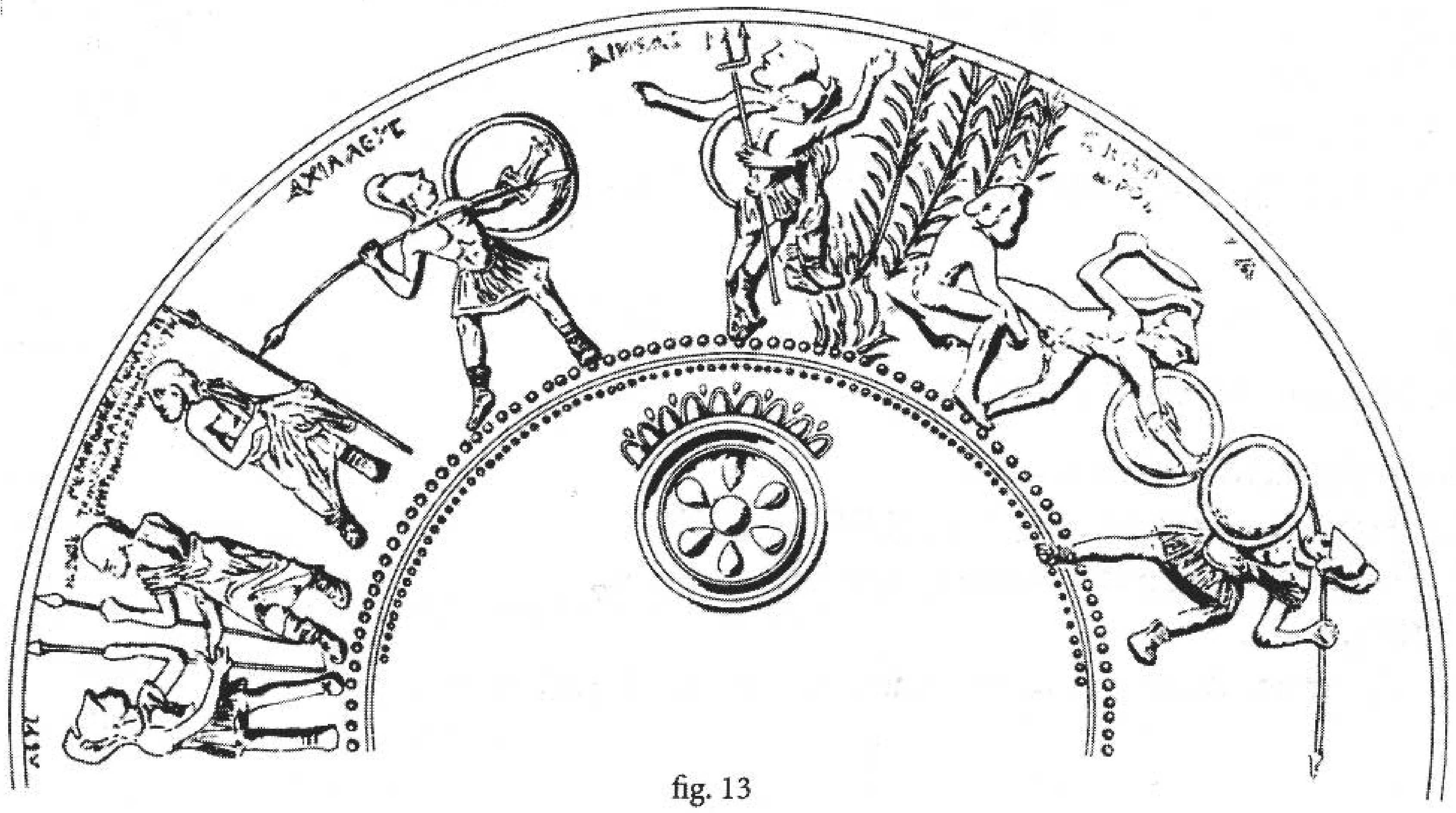


Planche VI
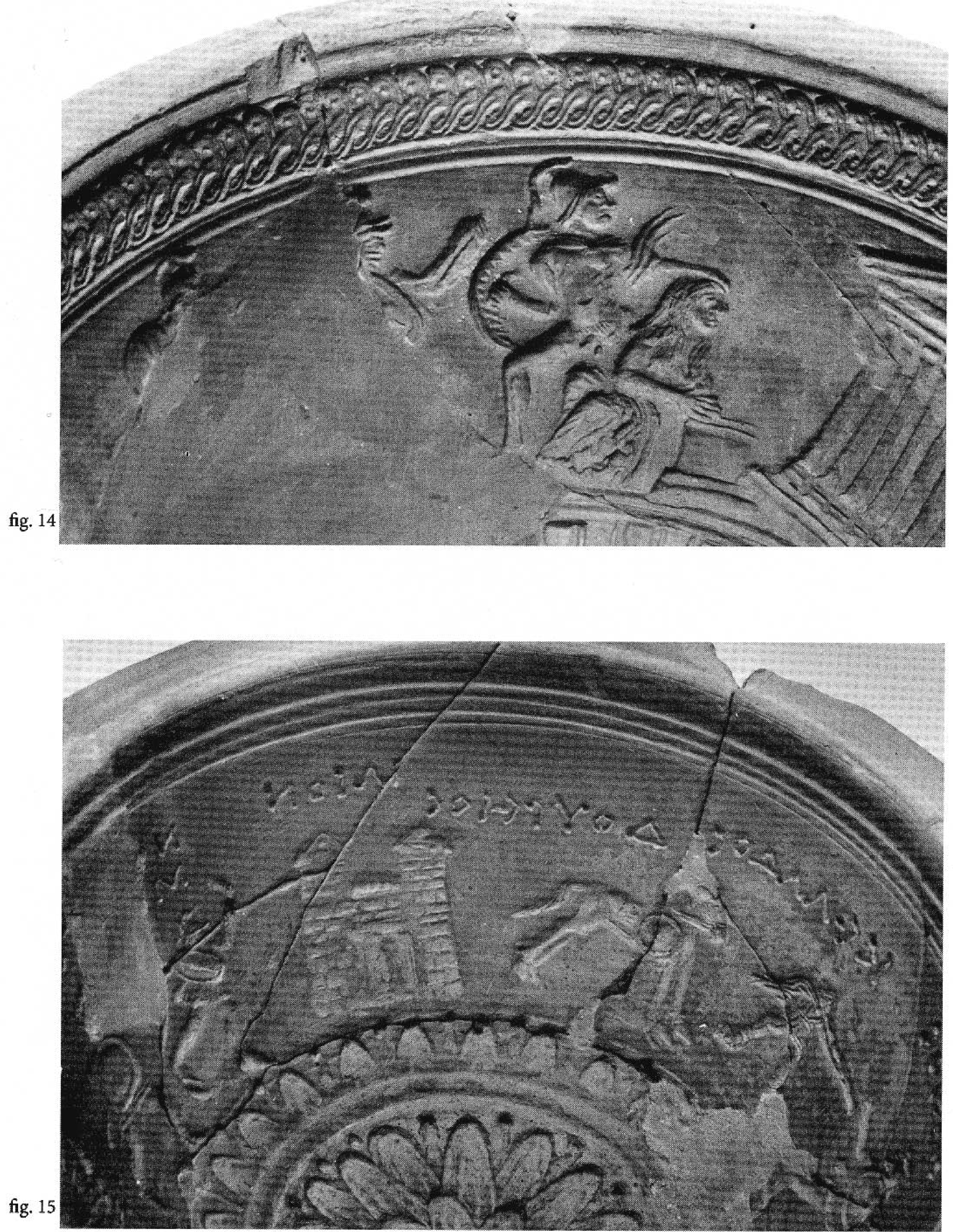
Planche VII
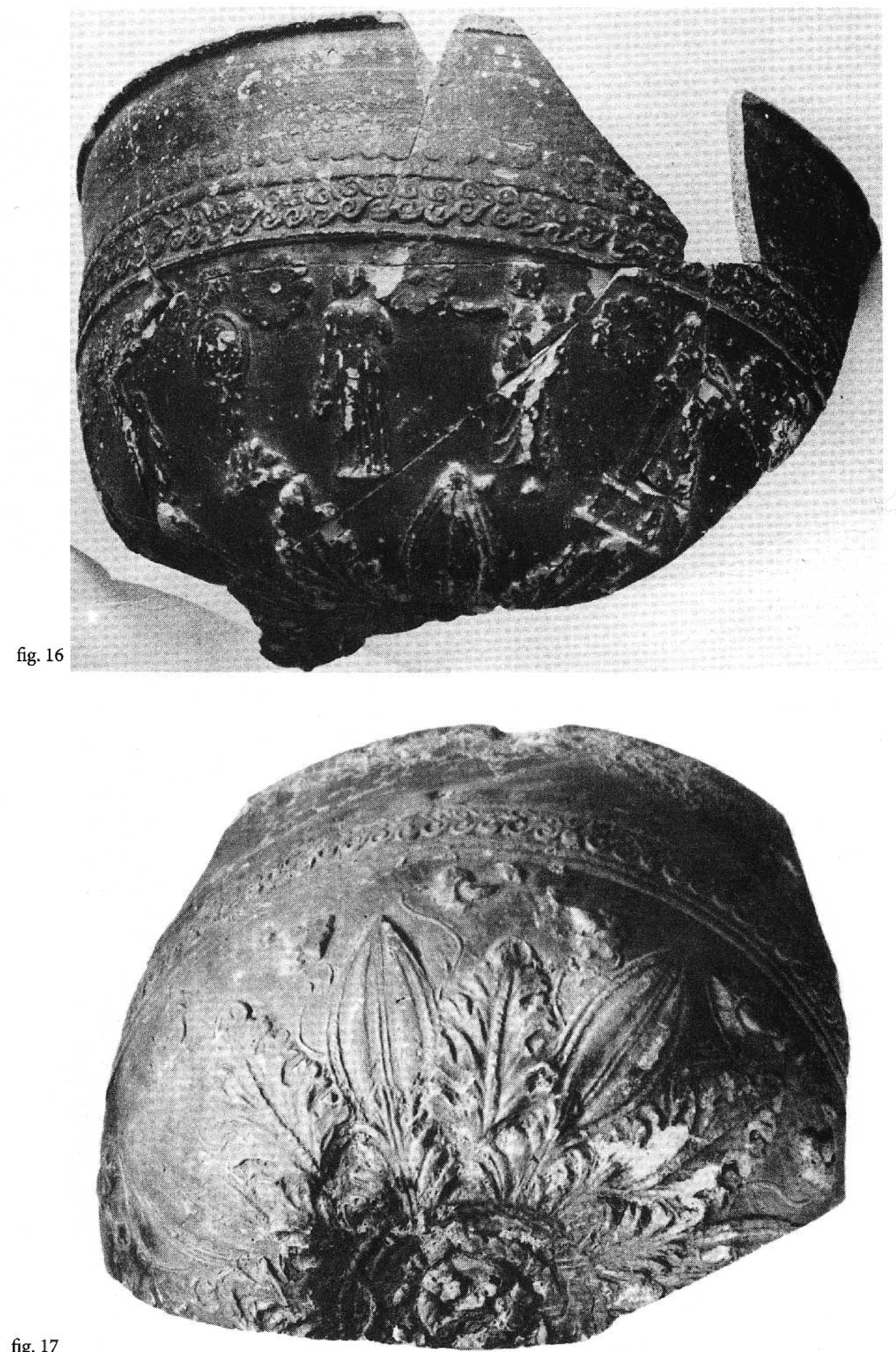
Planche VIII

fig. 18
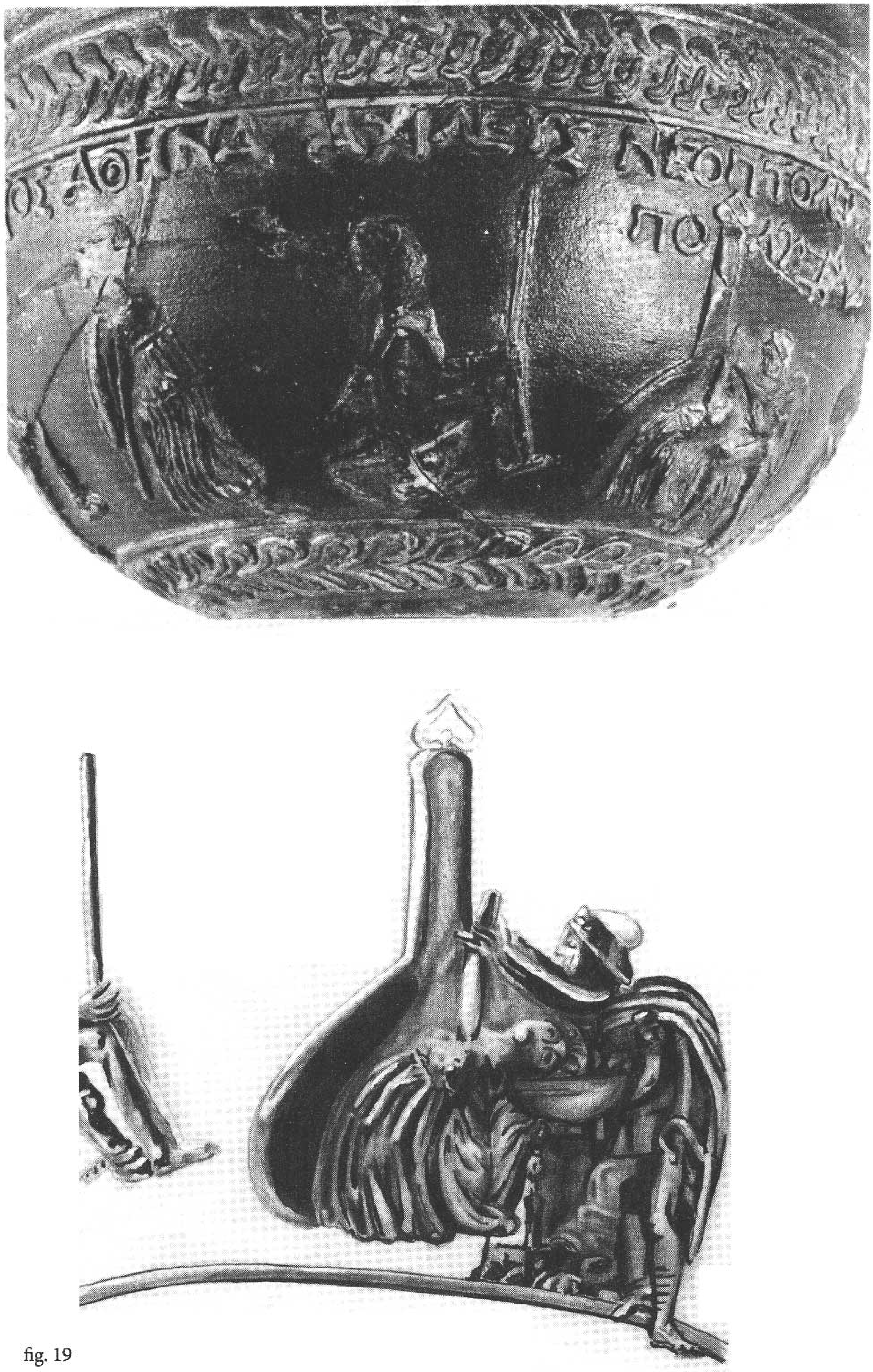